\title{
Integrating actor dynamics with land use cellular automata for modelling climate and environmental policy implementation at regional level
}

\author{
Dmitry V. Kovalevsky ${ }^{1}$, Richard J. Hewitt ${ }^{2}$ \\ ${ }^{1}$ Climate Service Center Germany (GERICS), Helmholtz-Zentrum Geesthacht (HZG), \\ Fischertwiete 1, 20095 Hamburg, Germany, \\ e-mail: dmitrii.kovalevskii@hzg.de \\ ${ }^{2}$ Information and Computational Sciences Group, The James Hutton Institute, \\ Cragiebuckler, Aberdeen AB15 8QH, Scotland, UK, \\ e-mail: richard.hewitt@hutton.ac.uk
}

\begin{abstract}
Successful implementation of environmental policies, including climate adaptation and mitigation policies, requires careful consideration of regional and local conditions. Consequently, there is growing understanding that regional models are needed to support climate and environmental policy making. Such models need to take into account the dynamics of geographical space as well as historic and expected future land use change patterns. One relevant geographical modelling approach is based on cellular automata (CA) which has a prominent track record of successful application to a diverse range of geographical problems. Traditionally, CA models are calibrated to reproduce the footprint of actor decision-making manifested in historical land use dynamics, and then projected forward to explore the effect of the observed dynamics on future periods. However, this is a poor representation of the way the world actually works, since policy decisions reflect current needs and priorities, not historic ones. Such a model cannot help us understand how decision-making actors might respond spontaneously to emerging land use outcomes. For these reasons, we believe there is considerable scope for existing CA-based geographical models to be improved by introducing realistic representations of the dynamic behaviour of decision-making actors. We present a modelling approach which retains the well-attested benefits of CA land use models, but which allows greater flexibility in modelling the dynamic behaviour of actors for particular "policy driven" land uses. To implement our approach, we integrate the APoLUS model (APoLUS stands for Actor, Policy and Land Use Simulator) an open-source, multi-platform model based on geographical CA - with a system dynamics (SD) model describing the actor dynamics. The SD model is tailored to reproduce the dynamics of interaction (and possible conflicting interests) of a number of aggregate actors that might influence regional development in general and might affect (either in positive or in negative way) the implementation of policy under study in particular. In the present paper, we describe new developments in the actor dynamics model family, progressing beyond earlier work in three key ways: (i) incorporating the
\end{abstract}


possible 'regime shifts' that might be related, in particular, to election cycles; (ii) describing in more detail the economic drivers of actor dynamics; (iii) introducing the stochasticity in a SD model.

\section{Introduction}

Environmental policies, such as those related to climate mitigation and adaptation, need to take into account land use impacts at regional scales. For example, a global shift to renewable energies implies large scale deployment of solar parks or wind farms, which requires correspondingly large land areas. As land is a scarce resource in many regions, a problem that is exacerbated by climate change, competition arises between energy uses and other land uses, e.g. agriculture or forestry (Nonhebel 2005). Policy makers should look to identify regions where such competition is likely, and try to tailor the policy design process to avoid unintended consequences (see, e.g. Deal and Pallathucheril, 2009), and maximise compatibility and multi-functionality (de Boer et al., 2015, de Groot, 2006), by exploring the options for "win-win" solutions (Hasselmann et al., 2015).

In consequence, there is growing understanding that regional models are needed to support climate and environmental policy making. Such models need to take into account the dynamics of geographical space as well as historic and expected future land use change patterns. One relevant geographical modelling approach is based on cellular automata (CA). CA modelling has a prominent track record of successful application to a diverse range of geographical problems, like assessing the vulnerability of coastal areas and islands to climate change (Engelen et al 1997), developing scenarios to combat desertification (Kok and Van Delden 2009), understanding the dynamics of shifting cultivation (Wickramasuriya et al 2009), supporting stakeholder dialogue around protected areas management (Hewitt et al. 2014) among others. In a geographical cellular automaton, land use change is simulated by the iterative application of transition rules to a raster map of land uses, in which each grid cell is attributed to one of a predefined set of land use classes. The evolution of grid cells is determined by their transition potential, which is a function of the user-defined transition rules. The amount of change in each land use class at each model time step depends on the demand for that particular class, known as "land claims". For this reason, these models are known as "constrained" CA models (White et al 1997).

In traditional geographic cellular automata, the transition rules are calibrated on the basis of several reference maps of the same region for selected moments in time (see e.g. Clarke et al., 1996, Straatman et al., 2004, Al-Ahmadi et al., 2009, Van Vliet et al., 2016, Shadman et al., under review). If the dynamics of the calibrated model are able to reproduce these reference maps in the calibration period with sufficient accuracy, it is assumed that the model will also perform well in making the projections beyond the calibration period. Such a model can usefully inform about the potential future consequences of past land use change tendencies.

To what extent does this modelling approach reflect the decision-making of actors affecting regional dynamics? 
Firstly, a key limitation of CA models in this regard, e.g. SLEUTH (Clarke et al., 1996) or Metronamica (RIKS, 2012) is that while the land use transitions modelled are the outcomes of actor decisions, the actors themselves are not directly represented in the CA model. This is not a problem for the simulation of incremental growth, such as the evolution of a city, but it is a major limitation for strongly actor-driven land change processes, like renewable energy. The APoLUS model (Hewitt et al., 2015, Hewitt, 2018) was developed to address this limitation, by incorporating the interaction of decision-making actors into the land use transition rules. This model forms the basis for the developments presented in this paper.

A further problem is that the model is designed to reproduce the footprint of actor decisionmaking as manifested in historical land use dynamics. This premise is clearly untenable in cases where future decision-making cannot realistically be expected to follow historic pathways, e.g. in the case of renewable energy, where development must increase to meet climate goals. This problem is normally countered by translating known future policy targets or socioeconomic projections into estimates for future land use demand, and then running simulations for the new pathways. This modelling strategy has been a standard approach for many years (see, e.g. Engelen et al., 1997, Volkery et al., 2007, Li et al., 2017).

Yet this approach is also unsatisfactory, since it assumes that policy decisions follow static pathways. In fact, we would not expect decision-making actors to remain static as the land use pattern evolves - the decisions they make are necessarily linked to emerging patterns in the territory. So, just as land uses in the traditional CA model compete with other land uses dynamically throughout the simulation period, so must individual actors dynamically respond to the behaviour of other actors and to the consequences of their own decisions in the evolving landscape.

Not every aspect of actors' decisions can be expressed in the model, general tendencies can be perceived (e.g. to promote or control particular land uses through subsidy or regulation), but some driving factors for decision-making may be unknown or unknowable. These kinds of uncertainties can be modelled by introducing an element of stochasticity into the modelled actors' interactions.

Further, actors' decisions are subject to the political process, which is expressed, in many countries through elections that are periodically celebrated as part of a regular election cycle. This periodicity may give rise to dramatic or unexpected policy changes, with correspondingly important effects on land use policy.

For these reasons, we believe there is considerable scope for existing CA-based geographical models to be improved by introducing realistic representations of the dynamic behaviour of decision-making actors. In the following paper, we present a modelling approach which retains the well-attested benefits of CA land use models, but which allows greater flexibility in modelling the dynamic behaviour of actors for particular "policy driven" land uses. The model we present is less 'mechanistic' than traditional CA land use models, and, we believe, potentially more suitable for modelling the influence of key actors on regional land use dynamics. 
To implement our approach, we integrate a geographical cellular automata model with a system dynamics model describing the actor dynamics (Hewitt et al., 2017; Kovalevsky et al., 2017).

The system dynamics model is tailored to reproduce the dynamics of interaction (and possible conflicts of interest) of a number of aggregate actors that might influence regional development in general and might affect (either in positive or in negative way) the implementation of policy under study in particular. The state variables of the system dynamics models include actor state variables adopted from Participatory Contextual Interaction Theory (PCIT), the latter being a result of integration of two approaches: Contextual Interaction Theory (CIT) and Participatory Action Research (PAR) (see the references on CIT, PAR, and PCIT in (Kovalevsky et al., 2017)). These state variables include:

- motivation - the actor's degree of motivation to implement the modelled process for the relevant policy;

- cognition - the actor's degree of awareness and knowledge that enable them to implement the modelled process for the relevant policy goal;

- resources - the resources (monetary or otherwise) at the actor's disposal;

- $\quad$ power - the power of the actor with respect to other actors in the model;

- affinity - the degree to which the actor is sympathetic towards implementation of the modelled process for the relevant policy goal.

In our previous studies (Hewitt et al., 2017; Kovalevsky et al., 2017) we explored the impact of actor dynamics on the deployment of solar energy installations and related land use change in the Navarre region, Spain. The system dynamics model describing local actor dynamics was integrated with the APoLUS model (APoLUS stands for Actor, Policy and Land Use Simulator) (Hewitt et al., 2015, Hewitt, 2018). APoLUS is an open-source, multi-platform model based on cellular automata (freely downloadable from https://simlander.wordpress.com/apolus/) and designed within the R software environment (The R Project for Statistical Computing, URL: https://www.rproject.org/). Our previous work (Hewitt et al., 2017; Kovalevsky et al., 2017), was focussed around the development of a system dynamic model family for the actor dynamic module integrated with APoLUS. We explored several alternative configurations for the system dynamics model describing the actor dynamics, starting from simple linear models and then developing more complex nonlinear schemes.

In the present paper, we describe further developments in the actor dynamic model family, progressing beyond this earlier work in three key ways:

1) We incorporate in the actor dynamics model possible 'regime shifts' that might be related, in particular, to election cycles.

2) We also pay closer attention than previously to describing the economic drivers of actor dynamics. These enter the model through the resources actor state variables. While in our previous studies rather simple assumptions on actor dynamics have been made (scenarios of exponential growth or stagnation), now we propose more versatile schemes for resource dynamics modelling. 
3) In previous versions of our model, stochasticity was present only in the cellular automata transition rules; we now introduce stochasticity into the actor modelling scheme such that the dynamics of actor state variables are described by stochastic dynamic systems. We believe that stochastic actor dynamic modelling would allow us to better account for the uncertainty in actor decision-making.

The rest of the paper is organized as follows. Sec. 2 describes the assumptions behind and equations of a simple two-actor model, with its three subsequent modifications (describing regime shifts, heterogeneity of resource dynamics, and stochastic effects) considered in detail in Sec. 3. Discussion of the results is provided in Sec. 4 . Sec. 5 concludes.

\section{Modelling the dynamics of actor state variables}

\subsection{Model assumptions}

To avoid hiding the concepts behind unnecessary technical details, we illustrate the basic ideas of model family development on a simple two-actor model, similar in spirit to (but not identical with) the 'linear model with equal influence weighing' described in detail in (Kovalevsky et al., 2017). The model could be immediately generalized to an $n$-actor case (as was done in (Kovalevsky et al., 2017)), but, for the sake of simplicity, we stick to a lowdimensional case of just two interacting aggregate actors: a proponent and an opponent of policy implementation under study.

In the remainder of this paper we are focussed on the actor-dynamics module of the integrated model (see Sec. 1). The ways of linking the actor dynamic module to land use cellular automata are described in (Hewitt et al., 2017; Kovalevsky et al., 2017).

As mentioned in Sec. 1, the n-th actor (in our case, $n=1,2$ ) is described with the following actor state variables: motivation $M_{n}$, cognition $C_{n}$, resources $R_{n}$, power $P_{n}$, and affinity $A_{n}$. In the most general framework possible, all of these variables might be time-dependent and endogenous. However, in what follows below we deliberately consider, for illustrative purposes, a much simpler case and assume the following:

- Cognition is a constant parameter, and for both actors cognition is the same $\left(C_{1}=C_{2}=C^{*}\right.$; specifically, $C^{*}=1$ in the figures).

- Actor affinities are constant parameters of equal absolute value but opposite signs: specifically, the first actor is the proponent of the implementation of policy under discussion (positive affinity, $A_{1}=+A^{*}>0$ ), while the second actor is the opponent of policy implementation (negative affinity, $A_{2}=-A^{*}<0$ ). Specifically, $A^{*}=1$ in the figures.

- Actor power $\left(P_{n}\right)$ and resources $\left(R_{n}\right)$ are either constant parameters or exogenous scenario variables (specific scenarios for power and resources will be explicitly defined in Sec. 3 for each of model versions considered).

- Motivation $\left(M_{n}\right)$ is endogenous, its dynamics are described by Eq. (4) below. 
In line with the approach adopted in (Hewitt et al., 2017; Kovalevsky et al., 2017), the overall impact of actor dynamics on policy implementation, $\Phi$, is defined as a total of individual impacts,

$\Phi=\Phi_{1}+\Phi_{2}$

where

$\Phi_{n}=\left(M_{n}+C_{n}+R_{n}\right) P_{n} A_{n}$.

Depending on the sign of the affinity, individual impacts $\Phi_{\mathrm{n}}$ can be positive, zero, or negative. In general, the overall impact $\Phi$ can also be of any sign (dependent on the balance between actor state variables of proponents and opponents).

\subsection{Actor dynamics}

We now introduce and analyse the endogenous model for motivation dynamics. Following (Kovalevsky et al., 2017), we introduce the influence $E_{n}$ of the $n$-th actor on other actors dependent on actor state variables. Specifically, we adopt the following parameterization for influence:

$E_{n}=P_{n} R_{n} A_{n} M_{n}$.

The endogenous dynamics of actor motivation are governed by a differential equation

$(d / d t) M_{n}=b_{n}{ }^{M}\left(E_{1}+\ldots+E_{n}\right)$,

where the sum in brackets in the r.h.s. is the aggregate influence of all actors on the $n$-th actor, and parameters $b_{n}{ }^{M}$ quantify the inertia of aggregate influence.

In our two-actor model, $\mathrm{n}=2$. For illustrative purposes, we make another simplification and assume that the 'inertial' parameters are equal for both actors,

$b_{1}{ }^{\mathrm{M}}=\mathrm{b}_{2}{ }^{\mathrm{M}}=\mathrm{b}^{\mathrm{M}}$,

and that the initial conditions for motivations at $\mathrm{t}=0$ are identical, too:

$M_{1}(t=0)=M_{2}(t=0)=M^{*}$.

Then, for this particular case, the Cauchy problems for motivations $M_{1}(t)$ and $M_{2}(t)$ are identical, and therefore

$M_{1}(t)=M_{2}(t)=M(t) \quad$ at all $t$

where the dynamics of $M(t)$ is governed by the equation

$(d / d t) M=b^{M}\left(P_{1} R_{1} A_{1}+P_{2} R_{2} A_{2}\right) M$,

or, given that $A_{1}=-A_{2}=A *$ (Sec. 2.1),

$(d / d t) M=b^{M}\left(P_{1} R_{1}-P_{2} R_{2}\right) A * M$.

In accordance with Eqs. (1)-(2), the overall impact of policy implementation is

$\Phi=\left[\left(M+C^{*}\right)\left(P_{1}-P_{2}\right)+\left(P_{1} R_{1}-P_{2} R_{2}\right)\right] A^{*}$. 


\section{Introducing new dynamical effects in the modelling scheme}

We now consider three versions of the two-actor model described in Sec. 2.2 that provide deeper insights in regime shifts, heterogeneous resource dynamics and stochastic effects, respectively.

\subsection{Regime shifts}

Suppose that before the 'regime shift' moment of time $t_{0}$ actor power and resources are constant over time and identical:

$\mathrm{P}_{1}=\mathrm{P}_{2}=\mathrm{P}^{*}, \quad \mathrm{R}_{1}=\mathrm{R}_{2}=\mathrm{R}^{*} \quad$ at $\mathrm{t}<\mathrm{t}_{0}$.

Then the r.h.s. of dynamic equation (9) is equal to zero, and therefore the motivation remains unchanged and equal to its initial value $M^{*}$. The r.h.s. of Eq. (10) is equal to zero as well: the situation is 'antisymmetric', and proponent and opponent impacts annihilate.

Suppose now that at $\mathrm{t}=\mathrm{t}_{0}$ the 'regime shift' occurs: for instance, the elections lead to the increase of the proponent power, while the opponent power remains unchanged (Figure 1). In simulations shown in Figures 1-3, we make a specific assumption

$\mathrm{P}_{1}=2 \mathrm{P}^{*}, \quad \mathrm{P}_{2}=\mathrm{P}^{*} \quad$ at $\mathrm{t}>=\mathrm{t}_{0}$,

while retaining the same scenario for resources:

$\mathrm{R}_{1}=\mathrm{R}_{2}=\mathrm{R}^{*} \quad$ at $\mathrm{t}>=\mathrm{t}_{0}$.

In accordance with Eq. (9), motivation experiences exponential growth at $t>=t_{0}$ (Figure 2). Impact on policy increases stepwise from zero to a positive level at the 'regime shift' year $t_{0}$, and then continues growing exponentially (Figure 3). Therefore, a sudden increase of the proponent's power shifts the balance towards a positive growing impact on policy.

\subsection{Heterogeneous resource dynamics}

In our previous modelling work (Hewitt et al., 2017; Kovalevsky et al., 2017) we always assumed that resources of all actors either grow exponentially with the same rate (the 'growth' scenario) or remain constant (the 'stagnation' scenario). If resources of different actors experience different dynamic regimes, this might also lead to balance shifts. We now explore this effect of heterogeneity with our simple two-actor model.

We now assume that power of both actors remains constant and identical throughout the full period of simulations.

$\mathrm{P}_{1}=\mathrm{P}_{2}=\mathrm{P}^{*}$.

At $t<t_{0}$, the resources are also constant and identical:

$\mathrm{R}_{1}=\mathrm{R}_{2}=\mathrm{R}^{*} \quad$ at $\mathrm{t}<\mathrm{t}_{0}$. 
Therefore, at $\mathrm{t}<\mathrm{t}_{0}$ the situation is again balanced, exactly as in Sec. 3.1. The motivation remains constant, the overall impact on policy remains zero.

However, at $\mathrm{t}=\mathrm{t}_{0}$ the resources of the proponent start growing linearly, while the resources of the opponent remain unchanged at their previous level (Figure 4):

$\mathrm{R}_{1}=\mathrm{R}^{*}\left[1+\mathrm{r}\left(\mathrm{t}-\mathrm{t}_{0}\right)\right], \quad \mathrm{R}_{2}=\mathrm{R}^{*} \quad$ at $\mathrm{t}>=\mathrm{t}_{0} \quad$ ( $\mathrm{r}=$ const).

A simple analysis shows that in the r.h.s. of the dynamic equation (9) the factor before the motivation is now proportional to the time elapsed from the moment $t_{0}$. Integrating then Eq. (9) by separation of variables, we find that the motivation grows as exponent of square time at $\mathrm{t}>=\mathrm{t}_{0}$ (Figure 5 ). However, the nonlinearity of motivation growth, that is very rapid in long-term, is not manifested in overall impact on policy (Eq. (10)): although at $t>=t_{0}$ the overall impact is positive and is no longer zero, it experiences just linear growth (Figure 6).

\subsection{Stochastic dynamics}

So far, all modelling schemes developed by us to describe the actor dynamic were deterministic. Stochasticity (noise effects) might be introduced in the proposed modelling approach in different ways. Mathematically, this would mean shifting from deterministic to stochastic differential equations (SDE). Consider, again, one illustrative stochastic modification of the two-actor model.

Suppose that powers of two actors $\left(P_{1}(t), P_{2}(t)\right)$ experience strong antiphase stochastic oscillations around the same constant average level $\left\langle\mathrm{P}_{1}\right\rangle=\left\langle\mathrm{P}_{2}\right\rangle=\mathrm{P}^{*}$. Specifically, we assume that

$\mathrm{P}_{1}(\mathrm{t})=\mathrm{P}^{*}(1+\mathrm{pV}), \quad \mathrm{P}_{1}(\mathrm{t})=\mathrm{P}^{*}(1-p V)$,

where $p$ is the constant scaling factor and $V$ is a white noise of unit spectral density. Due to antiphase behaviour, the sum of powers remains deterministic and constant over time, $P_{1}(t)$ $+P_{2}(t)=2 P^{*}$. At the same time, the power ratio might fluctuate in very broad limits if the magnitude of power fluctuations is strong enough, and this is the case illustrated by a scenario of 'turbulent' political situation in Figure 7.

The resources are assumed to be constant over time and identical:

$\mathrm{R}_{1}=\mathrm{R}_{2}=\mathrm{R}^{*}$.

An analysis of the dynamic equation (9) shows that it now becomes a linear SDE with multiplicative white noise. The theory of such equations is well developed; we omit technical detail and just summarize that the average motivation in our particular case will remain constant over time, while its variance will be growing over time (see an example of three random realizations of motivation dynamics in Figure 8). The overall impact on policy (Figure 9) also experiences pronounced stochasticity, often changing its sign, and with a magnitude varying in broad limits. 


\section{Discussion}

The modified system we have described above permits the dynamic interaction of land use decision-making actors to be simulated in a more realistic way than in a conventional CAbased land use model. Most importantly, decision-making actors, linked in APoLUS to land uses they influence, are now capable of transformative innovations in response to a simulated situation, such as winning or losing an election. This is represented in our model by a regime shift from opposing to supporting a particular land use policy, or vice versa. For the case of renewable energy, such policies are well documented in both directions. In Denmark, the first government of Rasmussen brought renewables development to a halt by withdrawing policy support in 2001, but changing direction in 2007 after re-election, leading to further expansion of the sector (Eikeland and Indeberg, 2016). In Spain, a long historic trajectory of support to renewable energy, accompanies by enormous expansion in land area occupied (Díaz Pacheco et al., 2018) was brought to an end by a change of government (Alonso et al., 2016). Our model is now able to simulate such a shift.

Further, while an actor's resources (usually, but not necessarily, considered to be economic) clearly influence the extent to which such an actor will support a policy, previous CA modelling approaches did not take into account the possibility that a change in the balance of resources amongst a group of implementing actors could affect the balance of power between the actors, and thus, potentially, change the configuration of the system. This modification allows for the simulation of economic events like a recession, or a withdrawal of subsidies, to be simulated in terms of the effect of all decision-making actors in the land use system.

Lastly, by adding an element of stochasticity into the actor interaction system, the rather unrealistic determinism of earlier modelled systems (e.g. Kovalevsky et al., 2017, Hewitt et al., 2017) is improved. This stochastic interference introduced is not strong, it is likely to increase the potential that chance events may influence circumstances that are already favourable to one outcome or other, rather than tip the system over into a new regime. However, given the other modifications previously noted, together with the possibility of a strong stochastic event, such an outcome cannot be entirely excluded.

These three additions to our earlier work on dynamic actor interaction within CA land use models bring the whole modelled system (implementation of land use innovations in actor negotiated or contested contexts) much closer to a true dynamical system of the kind observed elsewhere, like stock markets, traffic flows, or weather systems.

We have considered only the simple example case of two actors (one proponent and one opponent of a particular land use policy) and as such our system is still a 'toy model' of actor dynamics rather than a system implemented in a practical case. 


\section{Conclusions}

In this paper we have argued that conventional cellular automata land use models have disadvantages which may make them unsuitable for modelling strongly actor influenced land use changes. These models tend to represent land use changes as incremental growth, often based on historical land use dynamics used to fit the model during the calibration phase. However, some forms of land use change are driven very strongly by decision-making actors, so a more realistic approach to simulation is required that takes into account the dynamical systems behaviour of land use agents. The obvious example of a strongly actor-influenced land use change is that of renewable energy implementation in response to climate mitigation targets. However, other strongly actor dependent land systems do exist, for example, agriculture, so this model has wider general relevance. We demonstrate our dynamical systems modelling approach for the simple example of a two-actor model, for which several modifications were developed and analysed, including the policy regime shift, the heterogeneous dynamics of actor resources, and the stochasticity in actor power balance. Without unnecessary technical complications, these examples illustrate the prospects for development of more comprehensive actor dynamics modules for linking with the land use cellular automata model APoLUS. Along the same line, more realistic actor dynamics models might be developed, that would include multiple actors and more sophisticated assumptions on the details of dynamics of actor state variables.

\section{Acknowledgements}

The authors wish to acknowledge the financial support received by the EU FP7 project COMPLEX (Grant Agreement No 308601), which provided the inspiration for the APoLUS model and subsequent developments, as well as the opportunity for the two authors to collaborate. 


\section{References}

Al-Ahmadi, K., See, L., Heppenstall, A., Hogg, J. (2009): Calibration of a fuzzy cellular automata model of urban dynamics in Saudi Arabia. Ecological Complexity, 6(2), 80-101.

Alonso, P.M., Hewitt, R., Pacheco, J.D., Bermejo, L.R., Jiménez, V.H., Guillén, J.V., Bressers, H., de Boer, C. (2016): Losing the roadmap: Renewable energy paralysis in Spain and its implications for the EU low carbon economy. Renewable Energy, 89, 680-694.

Clarke, K.C., Hoppen, S., Gaydos, L. (1996): Methods and techniques for rigorous calibration of a cellular automaton model of urban growth. In: Third international conference/workshop on integrating GIS and environmental modeling, Santa Fe, New Mexico.

Deal, B., Pallathucheril, V. (2009): Sustainability and urban dynamics: assessing future impacts on ecosystem services. Sustainability, 1, 346-362, DOI:10.3390/su1030346

de Boer, C., Hewitt, R., Bressers, H., Alonso, P.M., Jiménez, V.H., Pacheco, J.D., Bermejo, L.R. (2015): Local power and land use: spatial implications for local energy development. Energy, sustainability and society, 5(1), 31.

de Groot, R. (2006): Function-analysis and valuation as a tool to assess land use conflicts in planning for sustainable, multi-functional landscapes. Landscape and urban planning, 75(3-4), 175186.

Eikeland, P.O., Inderberg, T.H.J. (2016): Energy system transformation and long-term interest constellations in Denmark: can agency beat structure? Energy Research \& Social Science, 11, 164-173.

Engelen, G., Uljee, I., White, R. (1997): Vulnerability Assessment of Low-lying Coastal Areas and Small Islands to Climate Change and Seal Level Rise-Phase 2: Case Study St. Lucia. Report and SimLucia User Manual. Maastricht.

Hasselmann, K., Cremades, R., Filatova, T., Hewitt, R., Jaeger, C., Kovalevsky, D., Voinov, A., Winder, N. (2015): Free-riders to forerunners. Nature Geoscience, 8, 895-898, DOI:10.1038/ngeo2593

Hewitt, R.J. (2018): A short presentation of the Actor, Policy, and Land Use Simulator (APoLUS). In: Geomatic Approaches for Modeling Land Change Scenarios (pp. 475-480). Springer, Cham.

Hewitt, R., de Boer, C., Pacheco, J.D., Hernández Jiménez, V., Alonso, P.M., Román, L., van der Meulen, M. (2015): APoLUS model full system documentation. Project Report for EU FP7 Programme COMPLEX Project (deliverable 3.5). Available at: https://www.researchgate.net/publication/292047909 APoLUS model full system docume ntation (accessed: June 2019)

Hewitt, R., Kovalevsky, D.V., de Boer, C., Hasselmann, K. (2017): Modelling actors' influence on land use change: a dynamic systems approach. In: Bregt, A., Sarjakoski, T., Lammeren, R. van, Rip, F. (Eds.). Societal Geo-Innovation: short papers, posters and poster abstracts of the $20^{\text {th }}$ AGILE Conference on Geographic Information Science, Wageningen University \& Research, 09-12 May 2017, Wageningen, the Netherlands. ISBN 978-90-816960-7-4. Accessible through https://agile-online.org/index.php/conference/proceedings/proceedings$\underline{2017}$

Hewitt, R., Van Delden, H., Escobar, F. (2014): Participatory land use modelling, pathways to an integrated approach. Environmental Modelling \& Software, 52, 149-165. 
Kok, K., van Delden, H. (2009): Combining two approaches of integrated scenario development to combat desertification in the Guadalentin watershed, Spain. Environment and Planning B: Planning and Design, 36(1), 49-66.

Kovalevsky, D.V., Hewitt, R., de Boer, C., Hasselmann, K. (2017): A dynamic systems approach to the representation of policy implementation processes in a multi-actor world. Discontinuity, Nonlinearity, and Complexity, 6(3), 219-245, DOI:10.5890/DNC.2017.09.001

Li, S., Juhász-Horváth, L., Pedde, S., Pintér, L., Rounsevell, M.D., Harrison, P.A. (2017): Integrated modelling of urban spatial development under uncertain climate futures: a case study in Hungary. Environmental Modelling \& Software, 96, 251-264.

Nonhebel, S. (2005): Renewable energy and food supply: will there be enough land? Renewable and Sustainable Energy Reviews, 9(2), 191-201.

Pacheco, J.D., Hewitt, R., Díez, A.L., Antequera, P.D. (2018): Valoración de Bases de Datos de Usos de Suelo para la localización y distribución espacial de la energía solar y eólica en España. Investigaciones Geográficas, (56).

RIKS (2012): Metronamica Documentation. Available at:

http://www.riks.nl/resources/documentation/Metronamica\%20documentation.pdf (accessed: June 2019)

Shadman Roodposhti, M., Hewitt, R.J., Bryan, B.A. (under review): Towards automatic calibration of neighbourhood influence in cellular automata land-use models. Computers Environment and Urban Systems.

Straatman, B., White, R., Engelen, G. (2004): Towards an automatic calibration procedure for constrained cellular automata. Computers, Environment and Urban Systems, 28(1-2), 149170.

van Vliet, J., Bregt, A.K., Brown, D.G., van Delden, H., Heckbert, S., Verburg, P.H. (2016): A review of current calibration and validation practices in land-change modeling. Environmental Modelling \& Software, 82, 174-182.

Volkery, A., Ribeiro, T., Henrichs, T., Hoogeveen, Y. (2008): Your vision or my model? Lessons from participatory land use scenario development on a European scale. Systemic Practice and Action Research, 21(6), 459-477.

White, R., Engelen, G., Uljee, I. (1997): The use of constrained cellular automata for high-resolution modelling of urban land-use dynamics. Environment and Planning B: Planning and Design, 24(3), 323-343.

Wickramasuriya, R.C., Bregt, A.K., Van Delden, H., Hagen-Zanker, A. (2009): The dynamics of shifting cultivation captured in an extended Constrained Cellular Automata land use model. Ecological Modelling, 220(18), 2302-2309. 


\section{FIGURES}

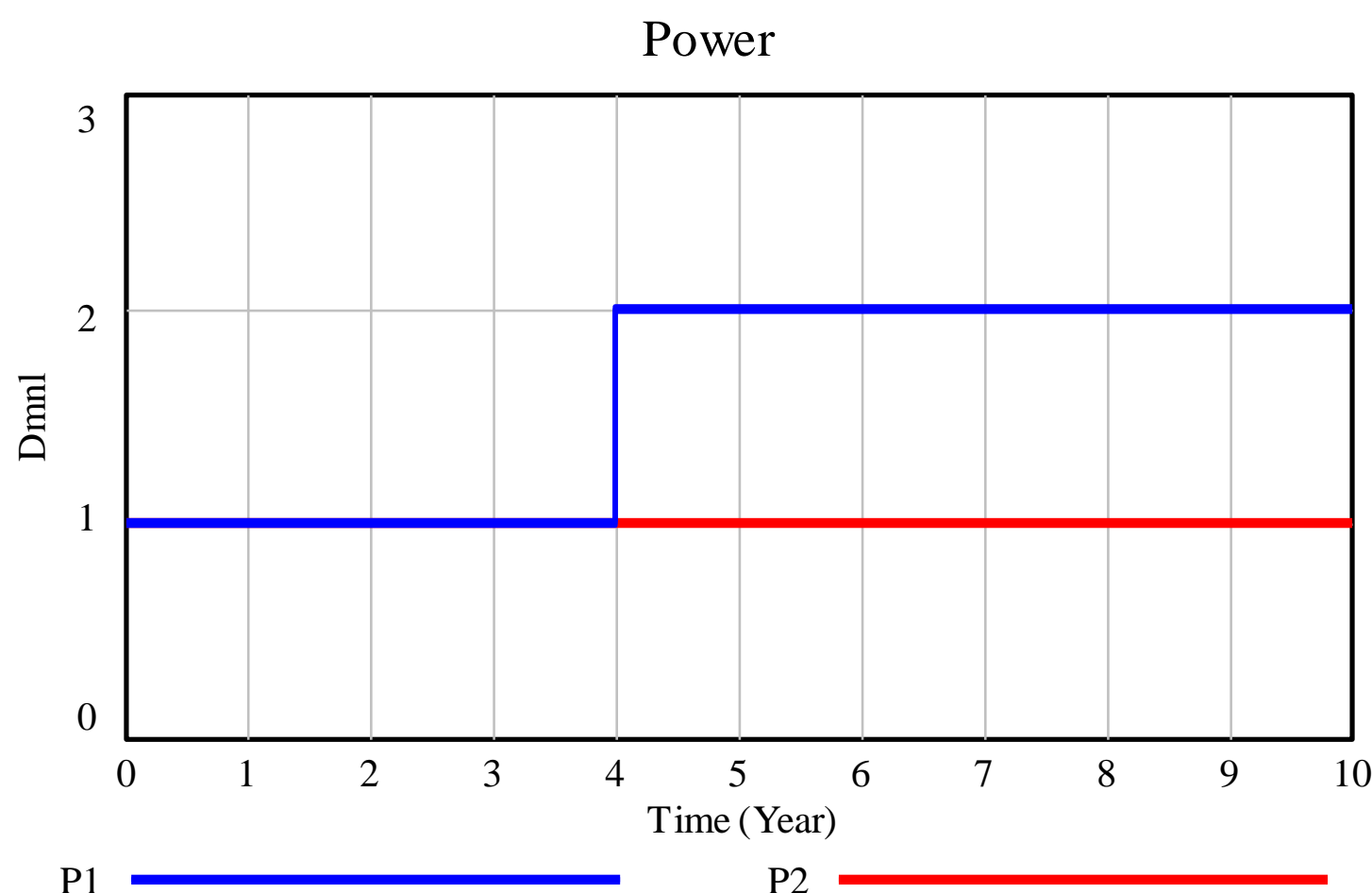

Figure 1: 'Regime shift' scenario: a stepwise increase of the power of the first actor (that has a positive affinity towards policy implementation) in a two-actor system at year $t_{0}=4$ 


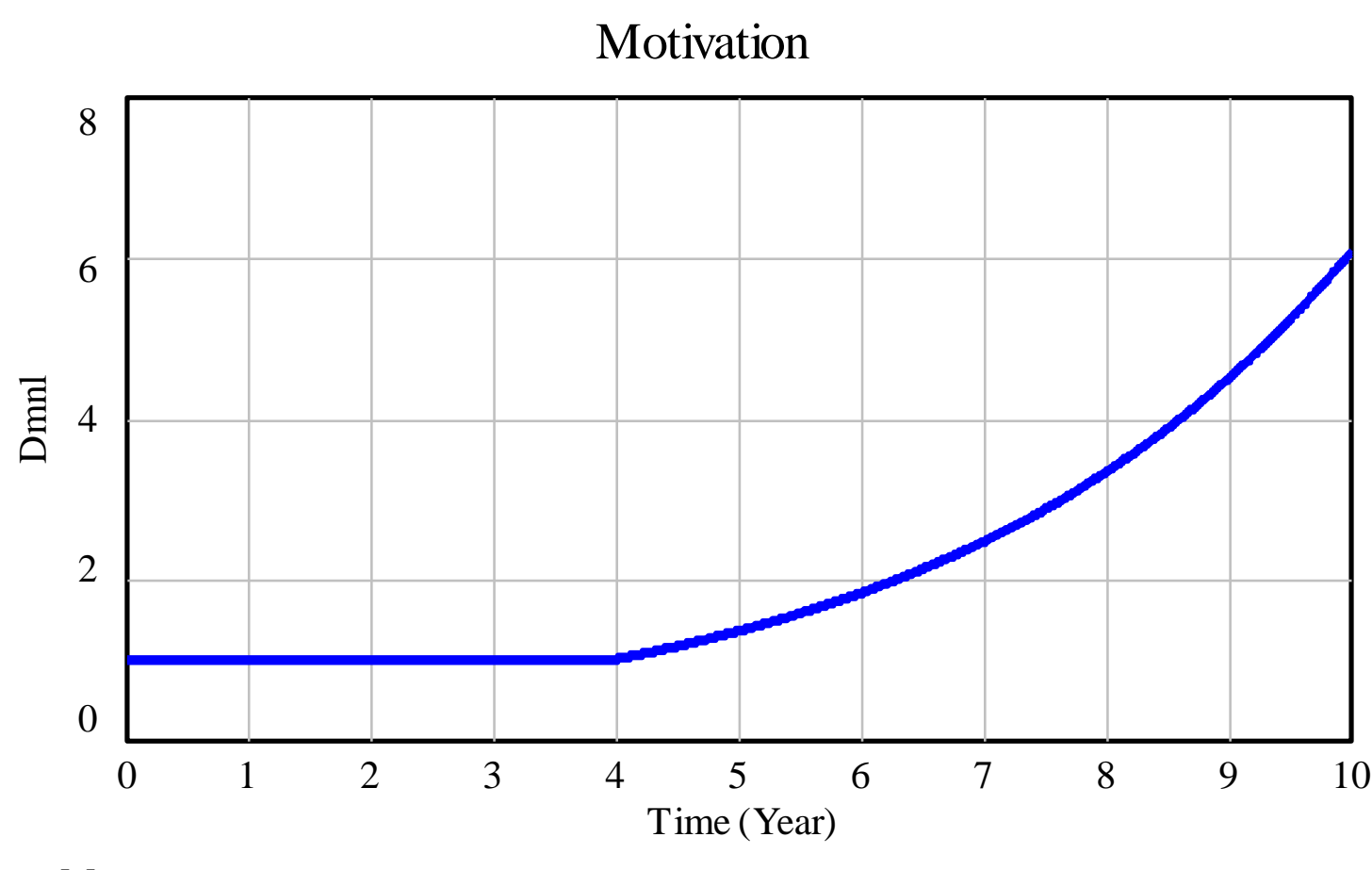

Figure 2: 'Regime shift' scenario: actor motivation starts increasing at year $t_{0}=4$, as a result of stepwise power increase of the proponent of policy implementation (see Figure 1) 


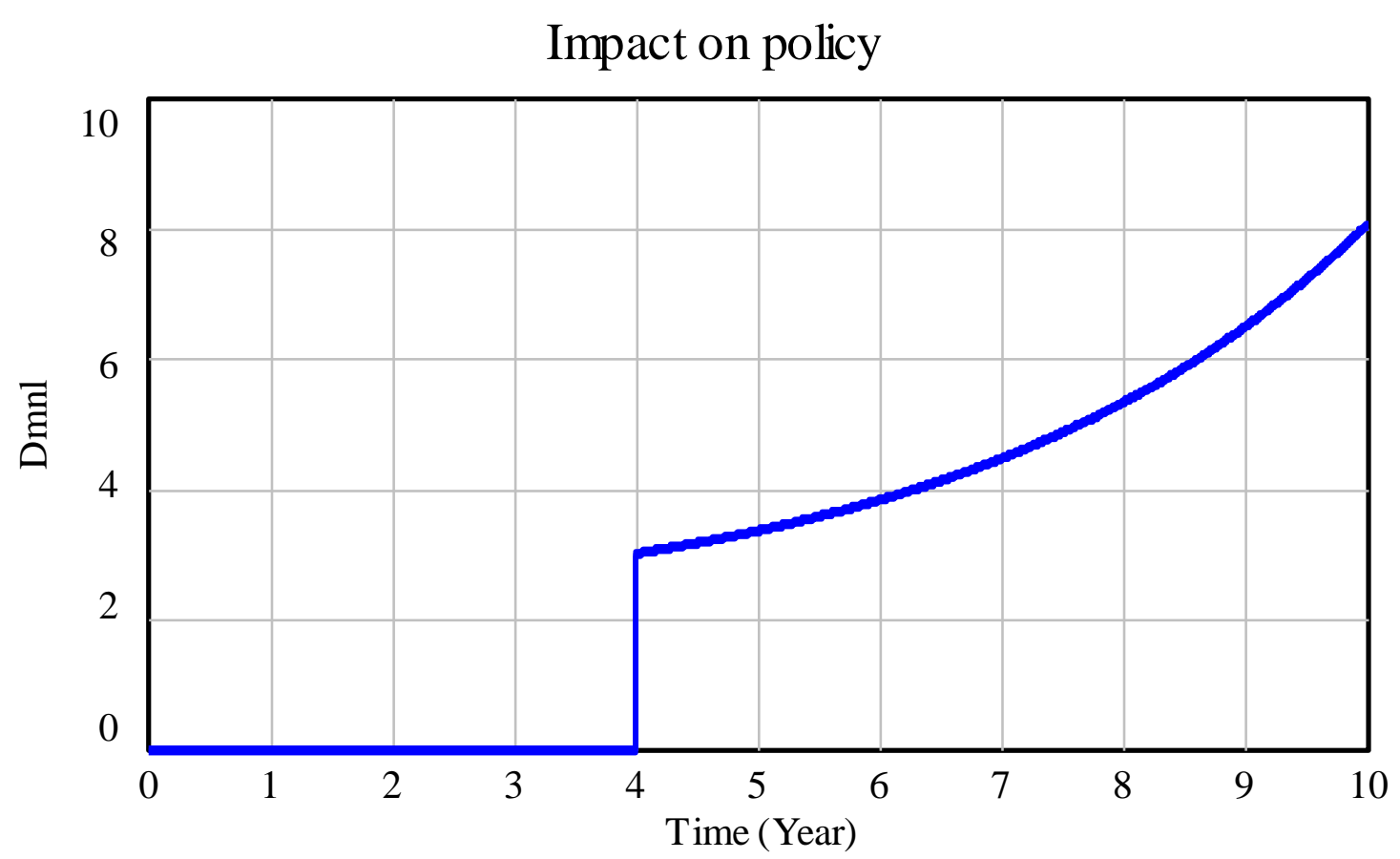

Imp act on policy

Figure 3: 'Regime shift' scenario: overall impact on policy increases stepwise from zero to a positive level at year $t_{0}=4$ and then continues to grow, as a result of stepwise power increase of the proponent of policy implementation at year $t_{0}=4$ (see Figure 1 ) 


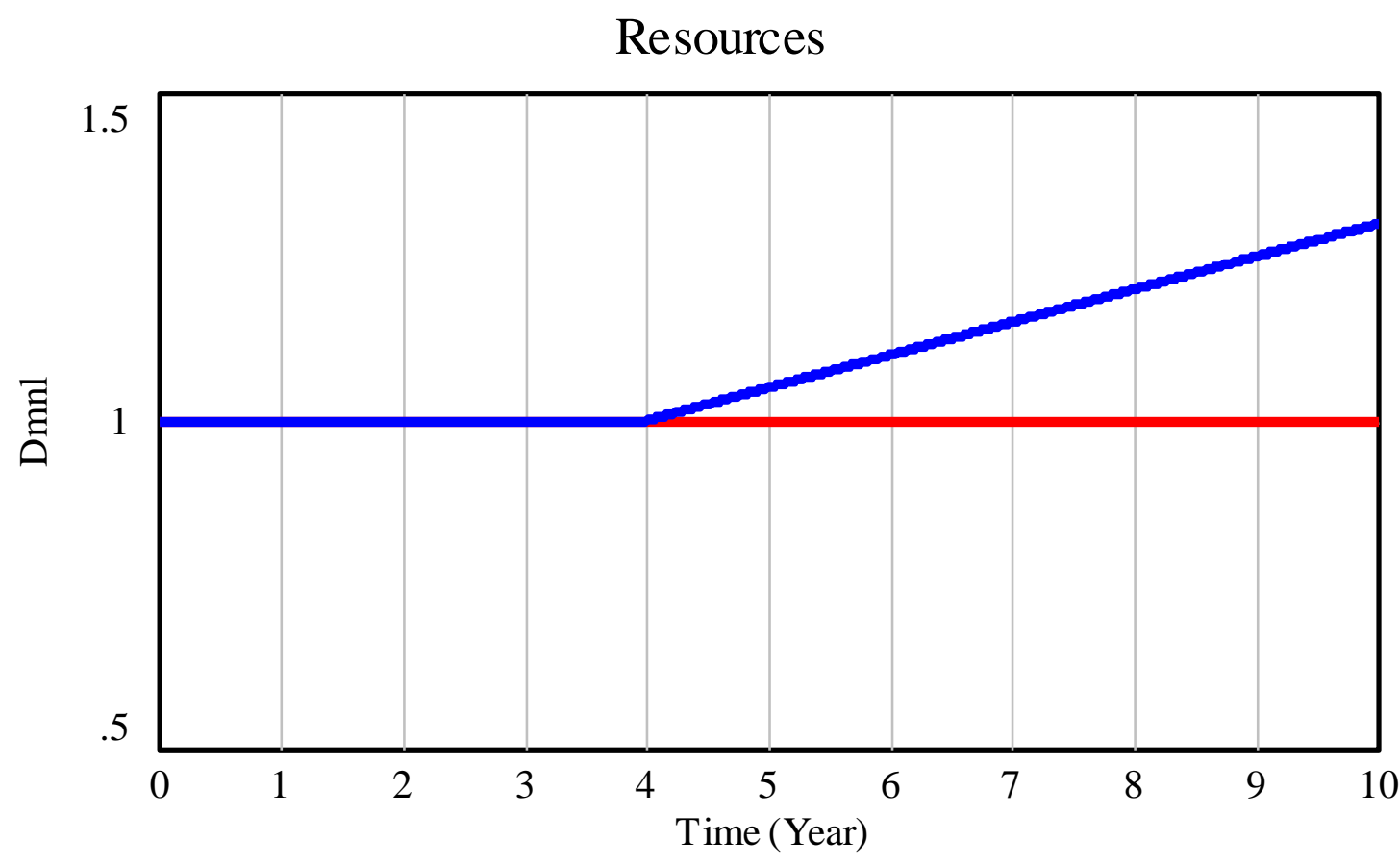

R1

R2

Figure 4: Differentiated resource growth scenario in a two-actor model: at year $t_{0}=4$ resources of the first actor start growing linearly, while resources of the second actor remain at previous constant level 


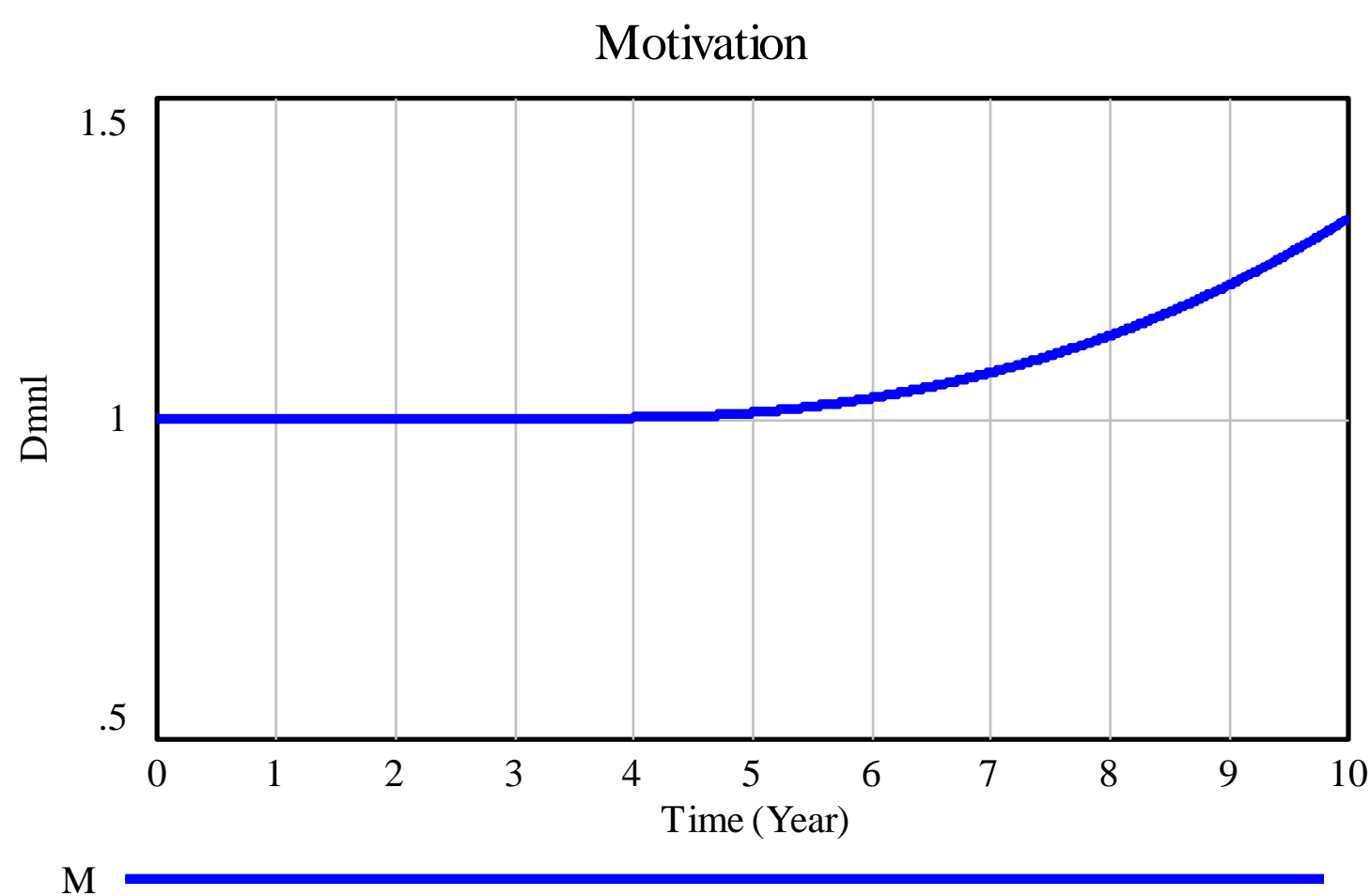

Figure 5: Differentiated resource growth scenario: actor motivation starts growing nonlinearly at year $t_{0}=4$ 


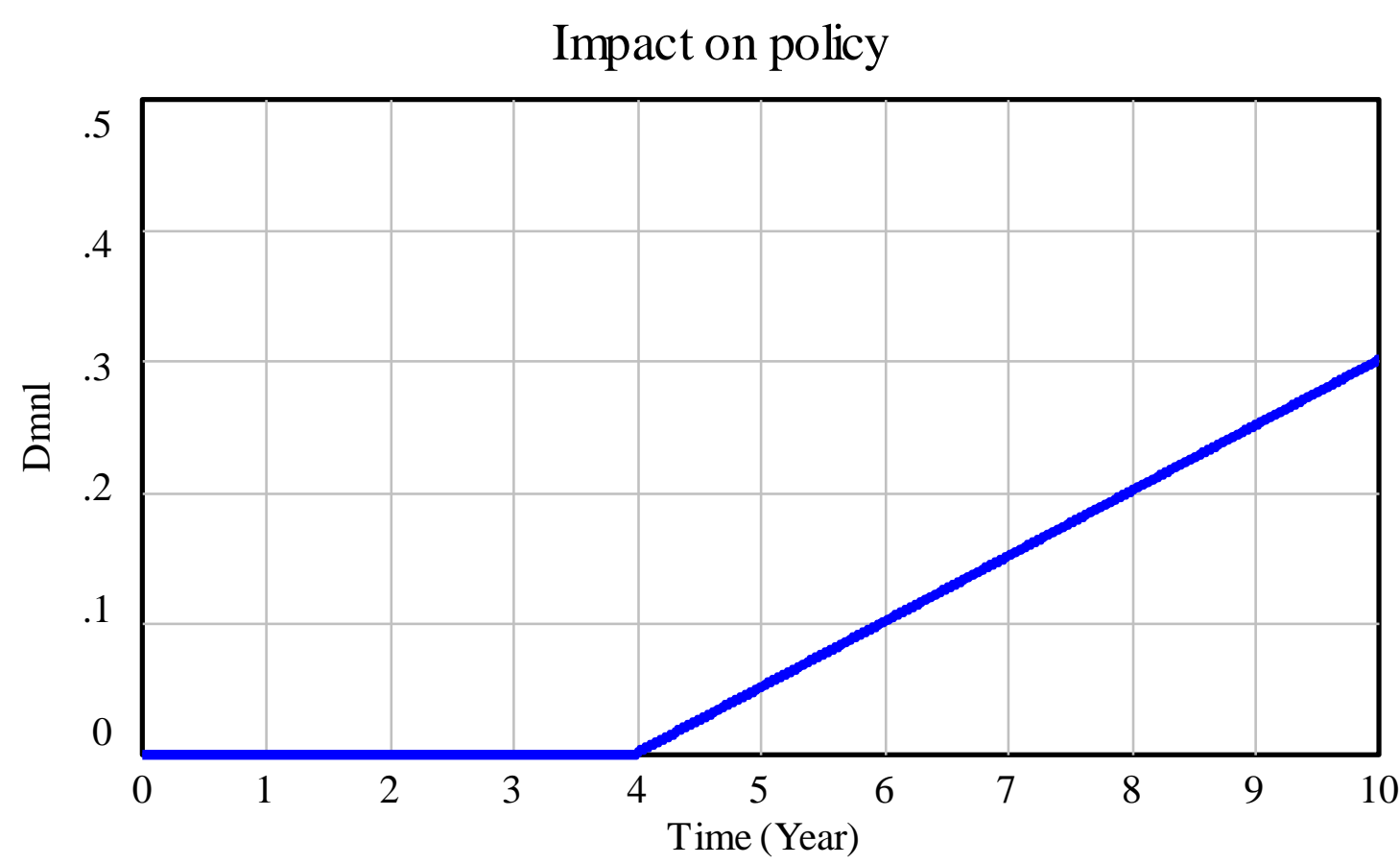

Imp act on policy

Figure 6: Differentiated resource growth scenario: overall impact on policy starts growing linearly from zero level at year $t_{0}=4$ 


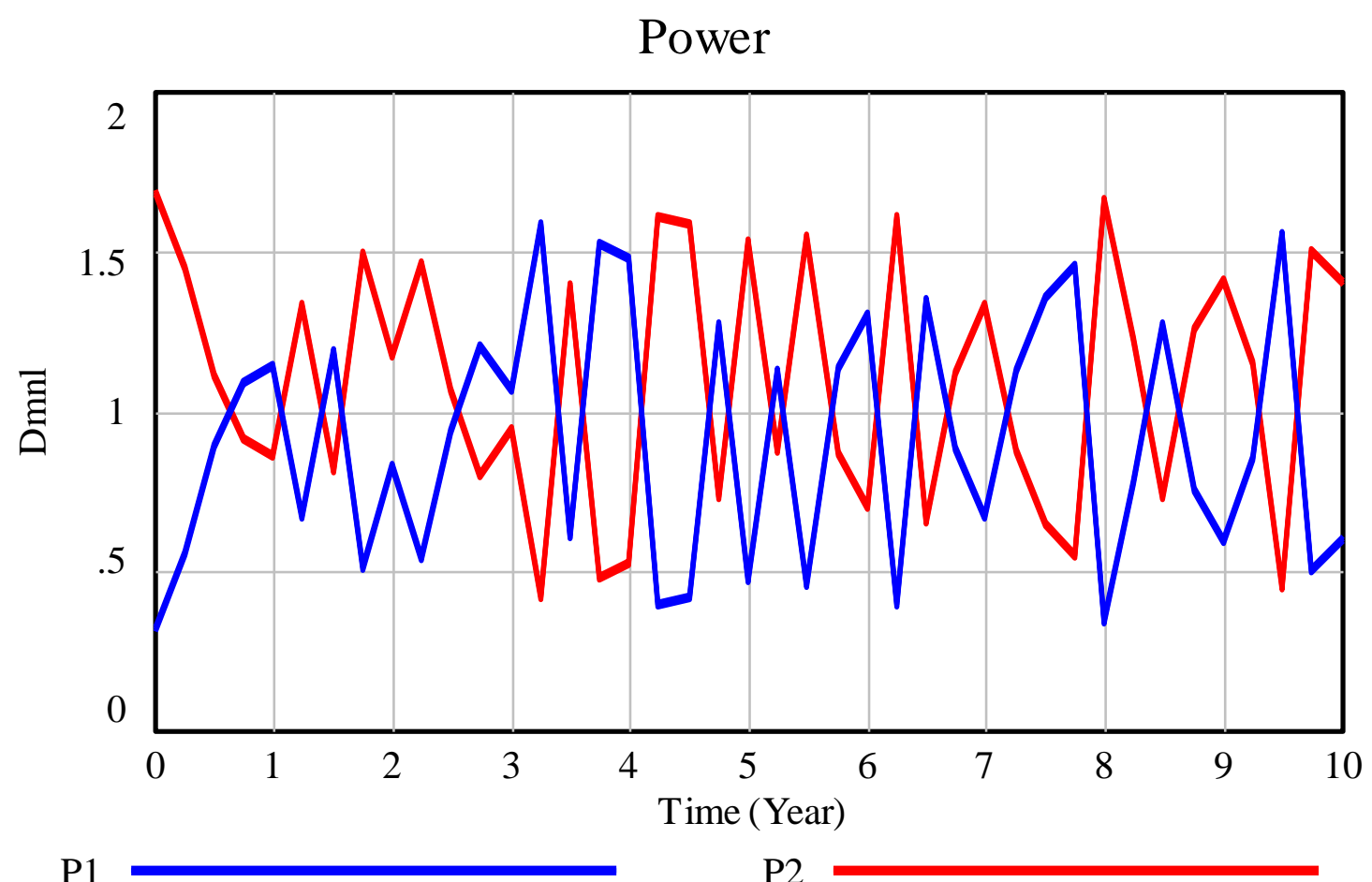

Figure 7: Stochastic dynamics of a two-actor system: powers of two actors $\left(P_{1}(t), P_{2}(t)\right)$ experience strong antiphase stochastic oscillations around the same average level $\left\langle\mathrm{P}_{1}\right\rangle=$ $\left\langle P_{2}\right\rangle=1$. The sum of powers remains constant over time, $P_{1}(t)+P_{2}(t)=2$ 


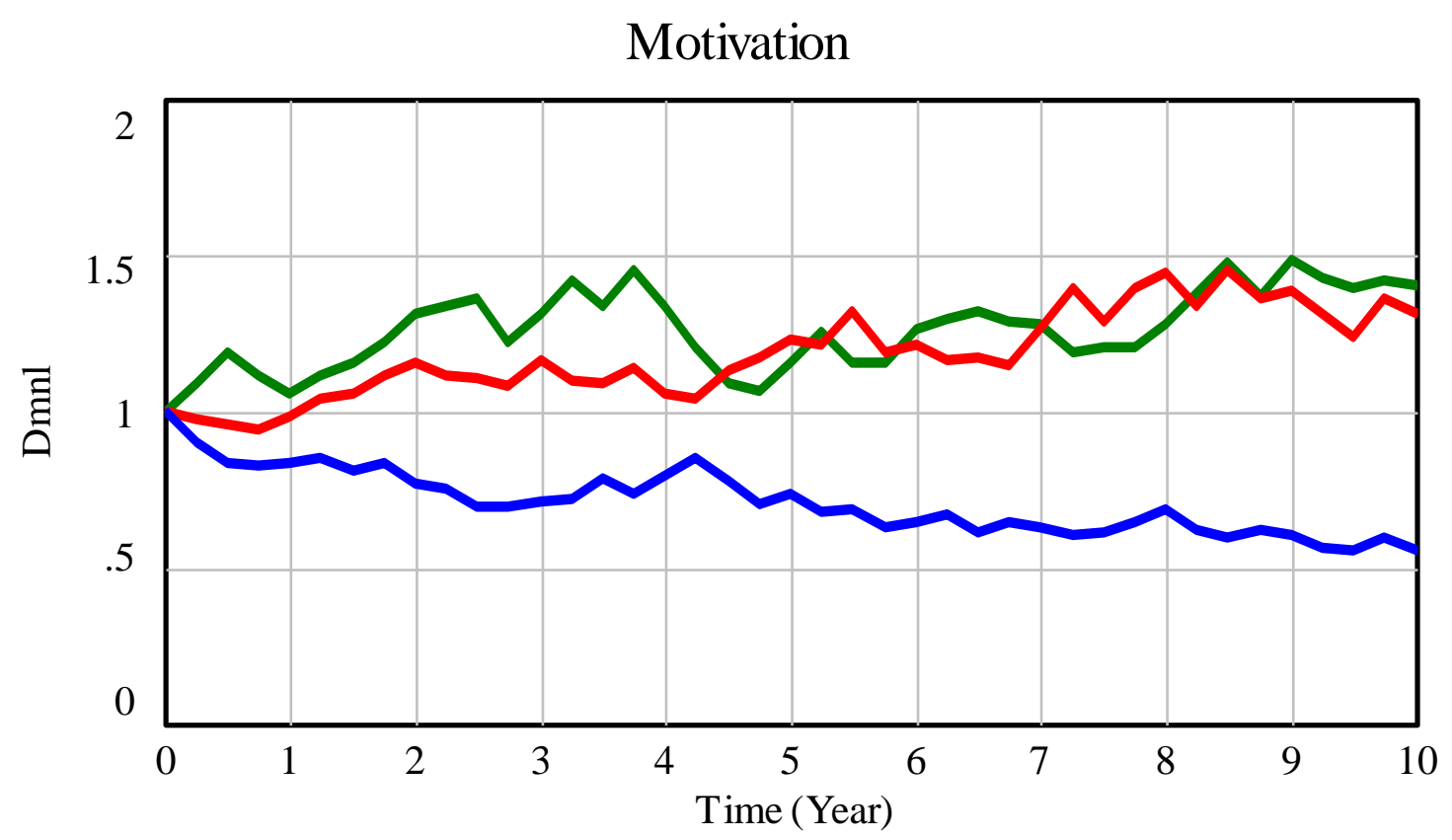

$\mathrm{M}$ (real. 1)

M (real. 3)

$\mathrm{M}$ (real. 2)

Figure 8: Stochastic dynamics of a two-actor system: three random realizations of actor motivation driven by antiphase stochastic oscillations of actor power (see Figure 7) 


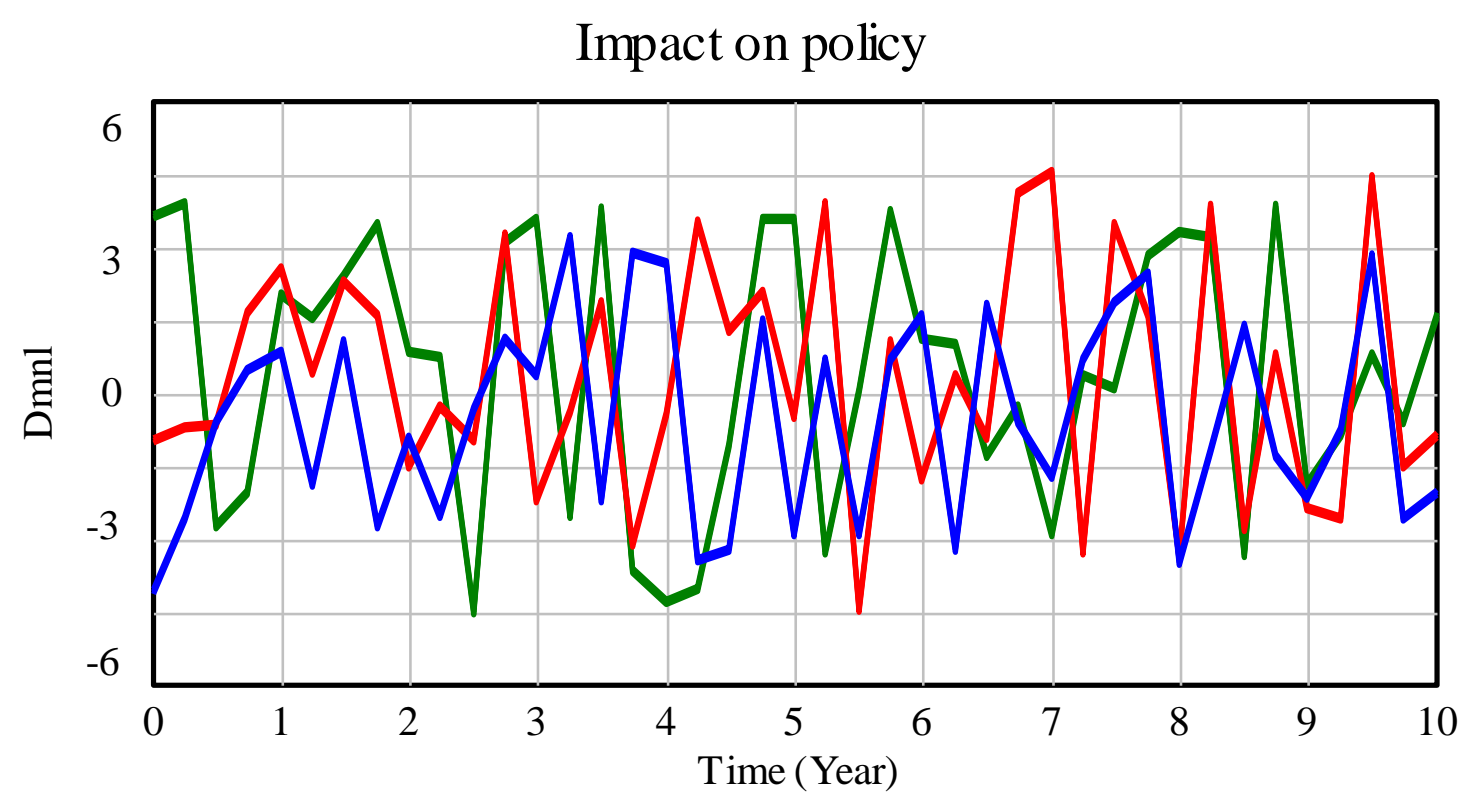

Imp act on policy (real. 1)

Imp act on policy (real. 2)

Imp act on policy (real. 3)

Figure 9: Stochastic dynamics of a two-actor system: three random realizations of overall impact on policy driven by antiphase stochastic oscillations of actor power (see Figure 7) 